Mediterránea Ser. Biol. (1990), n.o 12. Pág. 113-138

\title{
EL GÉNERO CHEILOSIA MEIGEN, 1822, EN LA CORDILLERA CANTÁBRICA (Diptera, Syrphidae) (*)
}

\author{
por \\ M. ${ }^{a}$ ÁNGELES MARCOS-GARCÍA (1)
}

\section{RESUMEN}

Se estudia un total de 42 especies del género Cheilosia, de las cuales tres: Cheilosia faucis Becker, Cheilosia gagatea Loew y Cheilosia ahenae Von Roser, son nuevas para la entomofauna ibérica. Se confirma la presencia en la misma de Cheilosia latifacies y se amplía el conocimiento de la distribución geográfica de las especies de este género, muchas de ellas citadas hasta el momento de una única localidad española. Se indican las especies vegetales sobre las que se han realizado las capturas, así como el nivel altitudinal y sector fitogeográfico en el que estas especies se han capturado con preferencia dentro de la provincia fitogeográfica Orocantábrica. Se representa por vez primera la estructura genital masculina de Cheilosia faucis.

\section{SUMMARY}

The genus Cheilosia Meigen, 1822 in the Cantabrian Cordillera (Diptera, Syrphidae). A total of 42 species of the genus Cheilosia is studied, of which three: Cheilosia faucis Becker, Cheilosia gagatea Loew and Cheilosia ahenea Von Roser are novel in the iberian entomofauna. The presence in the Iberian entomofauna of Cheilosia latifacies is confirmed and further contributions are offered to knowledge about the geographical distribution of this genus, many of them cited until now at a single site in Spain. The plant species on which the captures were made are described, together with the height above sea level and the phytogeographical sectors in which these species are mainly captured within the Orocantabrica phytogeographic province. The male genital structure of Cheilosia faucis is represented for the first time.

PALABRAS CLAVE: Cheilosia, Syrphidae, Diptera, nuevas citas, distribución espacial y temporal, Cordillera Cantábrica, España.

KEY WORDS: Cheilosia, Syrphidae, Diptera, new cites, space and temporal distribution, Cantabrian Cordillera, Spain.

$\left(^{*}\right.$ Este trabajo ha sido subvencionado por la C.A.I.C.T.Y.T. en el contexto del Proyecto de Investigación n. ${ }^{\circ}$ PR84-0921-C02-02.

(1) Departamento de Biología Animal y Pasasitología. Facultad de Biología. Universidad de Salamanca. 37071 - Salamanca (España). 


\section{INTRODUCCIÓN}

La Cornisa Cantábrica es uno de los enclaves ibéricos que hasta el momento han permanecido libres de estudios sirfidofaunísticos previos destinados a un conocimiento concreto y profundo sobre estos dípteros polinizadores. Sólo se conocen aportaciones aisladas de diversos autores extrajeros (LUCAS, 1976; THOMPSON, 1986, y VAN DER, GOOT 1958), donde siempre se pone de manifiesto el gran interés faunístico de esta cadena montañosa. Su especial situación geográfica, que hace que confluyan en ella tanto especies de distribución mediterránea como eurosiberiana, la gran riqueza de medios que alberga y su diversidad climática, hacen que a priori se presente como un área de especial interés desde el punto de vista entomológico, hecho que ha quedado claramente confirmado tras el presente estudio.

De las 170 especies de sírfidos que se han estudiado en la Cordillera Cantábrica (de un total de 294 que se conocían en la España peninsular), 42 pertenecen al género Cheilosia Meigen, 1822, siendo importante destacar que hasta el presente sólo 36 especies de este género estaban citadas de la Península Ibérica y solamente tres de ellas no han sido encontradas en dicha cordillera debido probablemente al hecho de que no rebasen el límite occidental de la Cordillera Pirenaica.

De las 42 especies estudiadas del género Cheilosia, ocho han resultado ser novedad para la entomofauna ibérica (MARCOS-GARCÍA, en prensa, a) y dos nuevas especies para la ciencia (MARCOS-GARCÍA, en prensa, b, y MARCOS-GARCÍA C. CLAUSSEN, 1989).

Las larvas de los representantes de este género holoártico presentan hábitos fitófagos y fungívoros, dándose en la mayoría de los casos especificidad alimentaria con respecto a la especie vegetal en la que se desarrollan. Los adultos, florícolas, suelen frecuentar las mismas plantas en las que se desarrollan sus larvas o bien visitar con cierta asiduidad determinadas especies vegetales (STUBBS \& FALK, 1983), de modo que se puede establecer cierta relación entre planta/especie Cheilosia.

\section{ÁREA DE ESTUDIO}

La provincia fitogeográfica Orocantábrica, establecida por Rivas-Martínez et al., 1984, comprende un amplio territorio ubicado en plena región eurosiberiana ibérica y ocupado por la sierras que constituyen la Cordillera Cantábrica y que se extienden de Occidente a Oriente desde el noreste de las provincias de Lugo y Orense hasta el suroeste del País Vasco, y de Norte a Sur, desde la parte meridional de Asturias y Cantabria hasta la septentrional de las provincias de León, Palencia y Burgos.

Se distinguen en ella cuatro pisos bioclimáticos: colino, montano, subalpino y alpino, aunque la mayoría de los ecosistemas pertenecen al piso montano.

Fitogeográficamente, Rivas-Martínez, et al., op. cit., la dividen en tres sectores y seis subsectores (Mapa 1). 


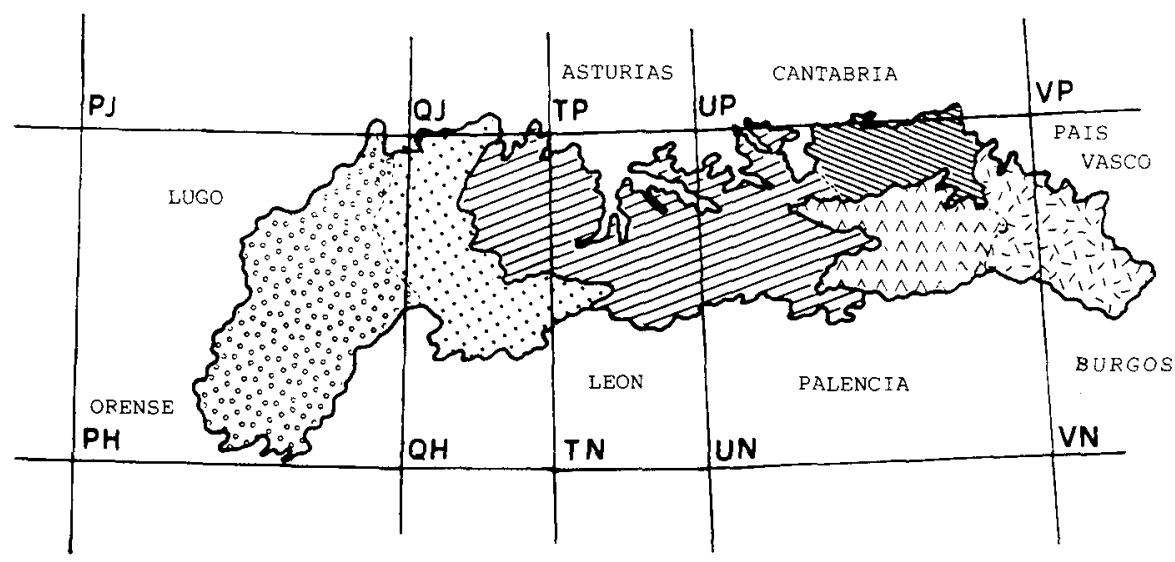

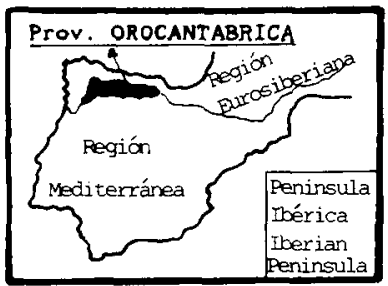

-A.

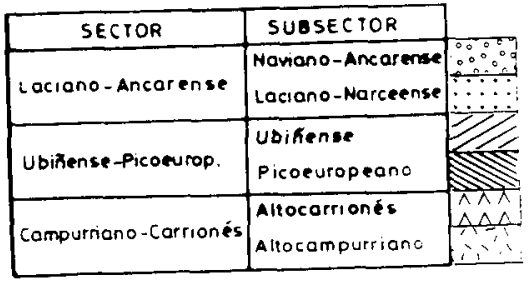

$\cdot$ B.

Mapa 1.-Mapa de la provincia fitogeográfica Orocantábrica: $\mathbf{A}$ = Situación de la Península Ibérica. $\mathrm{B}=$ Sectores y subsectores que la componen.

Map 1.-Map of the Orocantabrica phytogeographic province: $\mathrm{A}=$ Situation in the Iberian Peninsula. $\mathrm{B}=$ Sectors and subsectors comprinsing it.

\section{1.-Sector Laciano-Ancarense}

Situado en la parte más occidental, donde predominan los suelos silíceos y la precipitación estival alcanza sus niveles más bajos. Bioclimáticamente presenta pisos montano y subalpino. Se subdivide en dos subsectores: NavianoAncarense y Laciano-Narceense.

\section{2.-Sector Ubiñense-Picoeuropeano}

Ocupa una posición central. Se caracteriza por un predominio de suelos calcáreos y por presentar todos los pisos, desde el colino al alpino. Se distinguen en él dos subsectores muy semejantes entre sí: Ubiñense y Picoeuropeano.

\section{3.--Sector Campurriano-Carrionés}

En la sona oriental de la provincia Orocantábrica y caracterizado por tener suelos fundamentalmente silíceos, clima continental y una altitud media 
considerable. Se distinguen dos subsectores semejantes entre sí: Altocarrionés y Altocampurriano.

\section{MATERIAL Y MÉTODO}

El presente estudio se ha realizado en un total de 40 puntos de muestreo elegidos en base a sus características fitogeográficas y procurando que quedasen en ellos representados todos los tipos de vegetación existentes en la zona.

Las localidades fueron muestreadas con una periodicidad mensual a lo largo de tres años (1986, 1987 y 1988), y durante el período comprendido entre los meses de mayo a noviembre, dado que el resto del año las estaciones soportan bajas temperaturas y se encuentran en su mayor parte cubiertas de nieve.

En cada visita se prospectó durante una hora y se tomó nota de la planta o medio en el que se realizaban las distintas capturas.

Además de estas 40 localidades, han sido también prospectadas otras seis por su especial interés, aunque no de modo sistemático.

Al final, en un apéndice (Apéndice I) se incluyen las localidades de captura encuadradas en el sector fitogeográfico al que pertenecen. Se les ha asignado un número y su ordenación sigue la orientación oeste-este (Mapa 2). Además, se indica su altitud, provincia a la que pertenece, U.T.M. y dentro de cada localidad los distintos medios que se muestreaban en cada visita (diferenciados mediante una letra minúscula). En dicho apéndice también se encuadran las localidades no visitadas periódicamente (de la 41 a la 46).

Con el fin de conocer el piso bioclimático en el que las especies se distribuyen preferentemente, se han considerado cinco intervalos altitudinales correspondientes a los pisos de vegetación: colino, submontano, mesomontano, altimontano y subalpino, considerados por Rivas-Martínez et al., op. cit., aun-

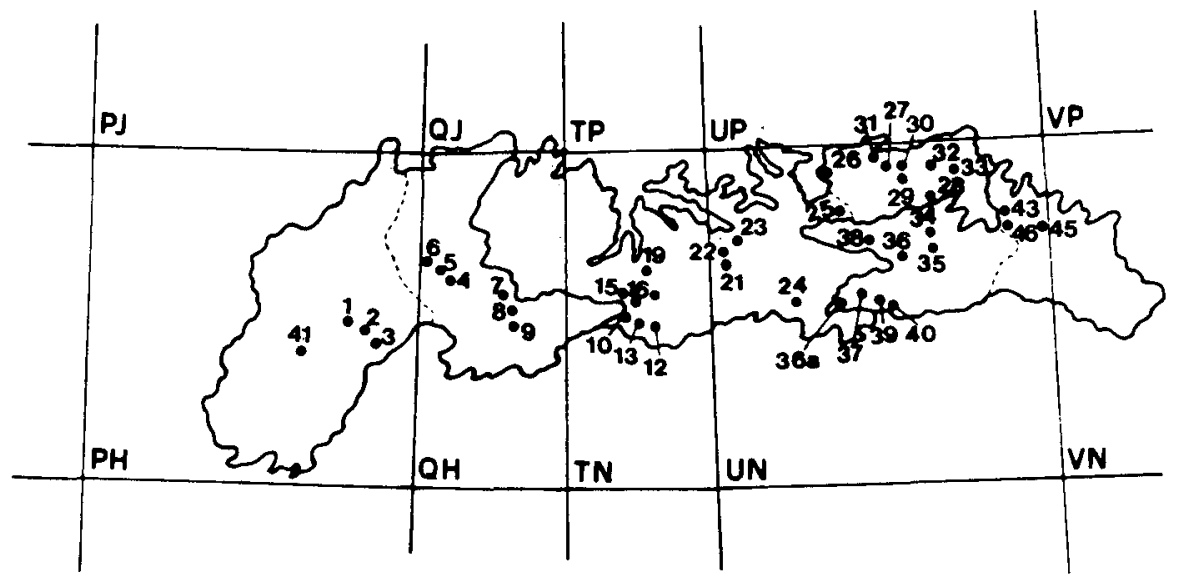

Mapa 2.-Mapa de la provincia fitogeográfica Orocantábrica. Estaciones de muestreo.

Map 2.-Map of the Orocantabrica phytogeographic province. Sampling sites. 
que con las particulares variaciones dependiendo de condiciones climáticas locales y sobre todo de su situación en umbría o solana. El número de localidades pertenecientes a cada intervalo es el siguiente:

\begin{tabular}{|c|c|c|c|}
\hline $\begin{array}{c}\text { PISO } \\
\text { BIOCLIMÁTICO } \\
\end{array}$ & $\begin{array}{l}\text { INTERVALO } \\
\text { DE ALTITUD }\end{array}$ & $\begin{array}{c}\text { NÚMERO } \\
\text { DE LOCALIDADES }\end{array}$ & $\mathrm{R}(\mathrm{K})$ \\
\hline Colino & 0-399 m.s.n.m. & 5 & 28 \\
\hline Submontano & 400-799 m.s.n.m. & 3 & 16 \\
\hline Mesomontano & 800-1.199 m.s.n.m. & 13 & 71 \\
\hline Altimontano & 1.200-1.599 m.s.n.m. & 12 & 72 \\
\hline \multirow[t]{2}{*}{ Subalpino } & $1.600-2.000$ m.s.n.m. & 7 & 41 \\
\hline & & 40 & $228=\mathrm{NR}$ \\
\hline
\end{tabular}

Las localidades de cada piso bioclimático son: colino $(26,27,31,32,33)$, submontano $(3,30,34)$, mesomontano $(2,7,9,10,12,13,20,24,29,35,37$, $39,40)$, altimontano $(4,8,14,16,17,18,19,21,22,25,36 \mathrm{a}, 38)$ y subalpino $(1,5,6,15,23,28,36)$.

Para realizar el cálculo de la distribución altitudinal de las especies cuyo número de datos obtenidos lo permitían, se ha utilizado el siguiente índice de frecuencias corregidas (DAGET et al., 1972; DAGET et GODRON, 1982).

$$
F e(K)=\frac{U(K) \cdot N R}{R(K) \cdot U(E)}
$$

Fe (K): Frecuencia corregida de la especie $\mathrm{E}$ en el intervalo altitudinal considerado (clase $\mathrm{K}$ ).

$U(K)$ : Número de muestras de la clase $K$ en las que está presente la especie $E$.

$R(K)$ : Número de muestras en la clase $K$.

$\mathrm{U}(\mathrm{E})$ : Número de muestras en las cuales la especie $\mathrm{E}$ está presente.

NR: Número total de muestreos $=228$.

Para el cálculo de la riqueza específica en cada uno de los subsectores fitogeográficos considerados se ha empleado un índice corregido en el que se tiene en cuenta el número de muestreos realizados en cada subsector:

$$
F c(K)=\frac{\text { NR.e }(K)}{M(K) \cdot E}
$$

e (K): Número de especies en el subsector considerado.

E: Número total de especies en el conjunto del área estudiada.

$\mathrm{Fc}(\mathrm{K})$ : Frecuencia corregida del número de especies en el subsector considerado.

$M(K)$ : Número de muestreos en el subsector considerado.

No se ha aplicado este índice al subsector Altocampurriano, dado que no se había realizado en él un número adecuado de muestreos. En el Apéndice II se presenta el listado de las especies estudiadas, donde se indica el número de ejemplares capturados en cada subsector fitogeográfico. 
A continuación se indica el material estudiado de cada especie, el intervalo estacional y altitudinal en el que se han encontrado, los subsectores fitogeográficos en los que se hallan presentes y las plantas sobre las que se realizaron las capturas.

No se indica el recolector del material por tratarse siempre de la autora del trabajo.

La ordenación de las especies se ha hecho de acuerdo con los cuatro grupos de especies que Sack, 1935, distingue en su obra.

\section{ESPECIES ESTUDIADAS}

\section{Grupo A (Ojos glabros)}

\section{Cheilosia ahenea Von Roser, 1840}

Esta especie se cita por vez primera en la Península Ibérica, por lo que se indican a continuación las características morfológicas de la misma:

Ojos glabros, genas sin largas setas, de coloración negra brillante, con bandas laterales de pulverulencia grisácea. Frente brillante, carente de pulverulencia. Tercer artejo antenal negro, a veces ligeramente anguloso en su extremo superior. Arista antenal negra con setas muy cortas. Mesonoto negro, brillante, con reflejos metálicos verdosos. Está cubierto por setas erguidas amarillogrisáceas, de longitud aproximadamente uniforme, aunque a menudo aparecen setas más largas de color negro o amarillo en los márgenes laterales o cerca del escudete. El margen posterior del escudete puede o no llevar macroquetas negras o amarillentas. Las patas son negras con setas amarillo-grisáceas. Abdomen negro-verdoso brillante, con setas blanco-amarillentas. Esternitos con pulverulencia grisácea que deja una zona brillante en el centro de extensión variable.

La genitalia y perfil epistomático se encuentran bien representados en SPEIGHT \& CLAUSSEN, 1987.

Esta especie vuela principalmente en el mes de junio en el área estudiada, ya que sólo un ejemplar ha sido colectado a principios de julio.

El $50 \%$ de los ejemplares fueron capturados sobre Caltha palustris L., y el otro $50 \%$ mangueando sobre vegetación herbácea. Todos ellos se colectaron en localidades montanas y subalpinas situadas entre 1.250 y $1.684 \mathrm{~m}$. de altitud, observándose clara preferencia por el nivel altitudinal subalpino.

Estos datos coinciden con los conocidos de países centroeuropeos, donde aparece preferentemente en antiguos pastos de montaña, pero contrastan con los del noreste de Irlanda, donde aparece principalmente en praderas calizas y formaciones de dunas situadas al nivel del mar (SPEIGTH \& CLAUSSEN, $o p$. cit.). Según estos autores, la planta huésped de $C h$. ahenea es probablemente de bajo porte y adaptada a crecer en pasos de montaña en los que se practica el pastoreo.

Se han capturados ejemplares de esta especie en localidades situadas en todos los sectores fitogeográficos, excepto en el Picoeuropeano. 


\section{DISTRIBUCIÓN GEOGRÁFICA:}

\section{Centroeuropea.}

Material estudiado: 1, 12-VI-86, 2000. 5a, 13-VI-86, 2oo; 1-VI-88, 10'. 6a, 13-VI-86, 40’0 y 1\%; 2-VI-87, 10’ y 1\%. 8b, 13-VI-86, 1\%; 1-VI-88, 10". 19, 14VI-86, 1ф; 4-VI-87, 2фᄋ. 21c, 3-VI-88, 10". 23, 16-VI-86, 3фo. 25, 6-VI-86, 1ф; 3-VI-88, 1․ 36a, 6-VI-88, 2o․ 36, 6-VI-88, 20\%".

Cheilosia cantabrica Marcos-García, en prensa, b

Esta especie, capturada en un canchal silíceo a 1.700 m.s.n.m., se describe en MARCOS-GARCÍA, en prensa, $b$.

\section{DISTRIBUCIÓN GEOGRÁFICA:}

Cordillera Cantábrica.

Material estudiado: 6c, 13-VI-86, $10^{\circ}$ y $2 \%$.

Cheilosia derasa Loew, 1857

Especie capturada en el piso subalpino volando en las cercanías de un regato de montaña. Es el único ejemplar de esta especie que se conoce en la Península Ibérica (MARCOS-GARCIA, en prensa, a). Es muy abundante en los Alpes a partir de los $1.200 \mathrm{~m}$. de altitud (GOELDLIN, 1974).

DISTRIBUCIÓN GEOGRÁFICA:

Alpes y Pirineos franceses.

Material estudiado: 5a, 2-VI-87, $10^{\circ}$.

Cheilosia faucis Becker, 1894.

Por ser nueva cita para la Península Ibérica, se indican a continuación las características morfológicas que la definen y se representa por vez primera su estructura genital masculina (Fig. 1).

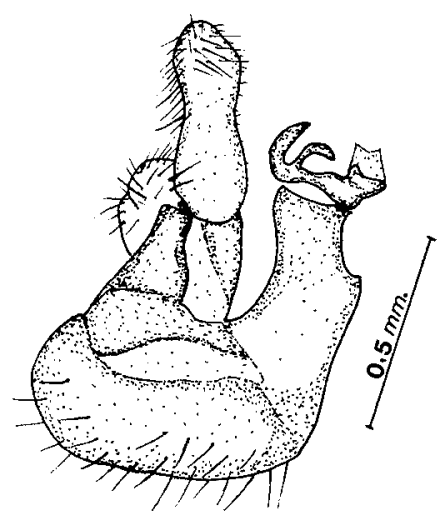

Fig. 1.-Estructura genital masculina de Cheilosia faucis Beck.

Fig. 1.-Male genitalia of Cheilosia faucis Beck. 
Frente negra con setas del mismo color. Tubérculo epistomático bien diferenciado y redondeado. Los dos primeros artejos antenales negros, el tercero de coloración más clara, tamaño negro y forma redondeada. Arista antenal claramente pubescente. Epistoma desnudo. Mesonoto mate con setas negras, erguidas y largas. Escudete con 6-8 macroquetas marginales negras y largas. Patas completamente negras. Alas grisáceas, más oscurecidas en su base. Escuamulas blanquecinas con el borde amarillento. Halterios amarillos con su extremo distal oscurecido. Abdomen negro y brillante, cubierto de setas blanquecinas excepto el borde posterior de los terguitos II, III y IV, que se encuentra cubierto de setas negras, cortas y reclinadas, formando a modo de bandas negras transversales abdominales.

\section{DISTRIBUCIÓN GEOGRÁFICA:}

Alpina.

Material estudiado: 5a, 1-VI-88, 10̛.

\section{Cheilosia gagatea Loew, 1857}

Es la primera vez que se cita esta especie en la Península Ibérica, por lo que se indican a continuación los caracteres morfológicos más significativos de la misma:

Epístoma lampiño y cubierto de dispersa pulverulencia blanquecina. Tubérculo epistomático tan prominente como el borde bucal. Antenas completamente negras, con su tercer artejo agudo en su extremo superior. Arista antenal con setas de mayor longitud que el diámetro de la misma. Setas del vértex y frente de color negro. Mesonoto cubierto de setas negras, de longitud uniforme y erguidas. Escudete con macroquetas en su margen posterior. Halterios amarillos y negros. Alas oscurecidas en su mitad basal. Abdomen negro, de bordes casi paralelos y con setas largas y erguidas de color blanco-amarillento.

El ejemplar fue capturado a $1.025 \mathrm{~m}$. de altitud sobre Heracleum spondylium L.

DISTRIBUCIÓN GEOGRÁFICA:

Europa Central y Meridional.

Material estudiado: 29, 5-VI-88, $10^{*}$.

\section{Cheilosia hercyniae Loew, 1857}

En España sólo se conocía esta especie del Pirineo Altoaragonés, por lo que se amplía su distribución hacia puntos más occidentales.

Se ha capturado a finales de primavera y en los meses de verano en altitudes comprendidas entre 600 y 1.550 m.s.n.m. sobre: Caltha palustris L., Heracleum spondylium L., y Tanacetum vulgare $\mathrm{L}$.

DISTRIBUCIÓN GEOGRÁFICA:

Europa Central y Septentrional.

Material estudiado: 5a, 13-VI-86, 1ㅇ. 12, 11-IX-87, 1․ 34, 22-VI-87, 1 ㅇ. $43,21-$ VII- 85,1 Q. 
Los datos anteriores son los segundos que se conocen sobre la presencia de esta especie en España, ya que únicamente se citó de Barcelona hace 60 años (GIL-COLLADO, 1930).

Los ejemplares han sido capturados siempre sobre Ranunculáceas ( $\mathrm{Cal}$ tha palustris L. y Ranunculus ollissiponensis Pers.) en los primeros días del mes de junio, tras desaparecer la nieve en los subsectores Laciano-Narceense y Ubiñense y en altitudes superiores a los 1.200 m.s.n.m.

\section{DISTRIBUCIÓN GEOGRÁFICA:}

Centroeuropea.

Material estudiado: 6a, 13-VI-86, 1\%; 2-VI-87, 19. 9d, 1-VI-88, 19. 19a, 2-VI-88, 2ơ.

\section{Cheilosia latifacies Loew, 1857}

Esta especie ha sido citada de España en diferentes ocasiones, aunque erróneamente, ya que el material sobre el que se basaron estas citas correspondía a Cheilosia paralobi Malski, 1962 (MARCOS-GARCÍA, 1987). Debido a la imposibilidad de estudiar la totalidad de los ejemplares sobre los que se citó esta especie por vez primera en España, no se pudo concluir la inexistencia de la misma en el ámbito peninsular. Así pues, los datos obtenidos confirman su presencia, al menos en la Cordillera Cantábrica.

Todos los ejemplares se han colectado sobre umbelíferas en localidades comprendidas entre 250 y $1.050 \mathrm{~m}$. de altitud y situadas en la mitad oriental de la provincia Orocantábrica, concretamente en los subsectores Picoeuropeano y Altocarrionés.

\section{DISTRIBUCIÓN GEOGRÁFICA:}

Paleártica.

Material estudiado: 30, 21-VI-87, 1o; 5-VI-88, 10; 10-IX-88, 2qQ. 33, 15 VII-87, 1․ 40, 23-VI-87, 1 ९.

\section{Cheilosia nasutula Becker, 1894}

Especie conocida en la Península Ibérica exclusivamente del Pirineo Altoaragonés. Ha sido capturada en el mes de junio a partir de $1.379 \mathrm{~m}$., siempre sobre Caltha palustris L. y en los subsectores Laciano-Narceense y Ubiñense.

\section{DISTRIBUCIÓN GEOGRÁFICA:}

Eurosiberiana.

Material estudiado: 6a, 1-VI-88, 19. 19a, 2-VI-88, 300 .

Cheilosia nigripes (Meigen, 1822)

Esta especie estaba ya citada de Huesca y Santander.

Los cuatro ejemplares se han capturado en el mes de junio sobre Ranunculáceas y en localidades altimontanas pertenecientes al subsector Ubiñense. 


\section{DISTRIBUCIÓN GEOGRÁFICA:}

Euroasiática.

Material estudiado: 13, 3-VI-87, 10 y 1\%; 2-VI-88, 10’. 17, 4-VI-87, 1 \%. Cheilosia pagana (Meigen, 1822)

Esta especie ya estaba citada de la franja norte peninsular.

Se ha capturado a $150 \mathrm{~m}$. de altitud, datos que coinciden con lo expuesto por GOELDLIN, 1974, quien la considera en Suiza presente principalmente en las zonas bajas.

\section{DISTRIBUCIÓN GEOGRÁFICA:}

Eurosiberiana.

Material estudiado: 26,6 -VII- $86,10^{\circ} .31,10-I X-88,1$ (q.

\section{Cheilosia paralobi Malski, 1961}

Esta especie, descrita de Argelia, se conocía en España de Granada, Murcia, Málaga y distintas localidades del Sistema Central (MARCOS-GARCÍA, 1987). Los datos anteriores representan las citas más septentrionales de su distribución.

Los dos ejemplares se han capturado en altitudes superiores a los 1.000 metros y en localidades pertenecientes al subsector Laciano-Narceense.

\section{DISTRIBUCIÓN GEOGRÁFICA:}

Iberomogrebí.

Material estudiado: 7, 1-VI-88, 19. 9, 7-XI-86, $10^{\circ}$.

Cheilosia personata Loew, 1857.

Esta especie en la Península Ibérica sólo se conocía del Pirineo oscense, de donde fue citada recientemente (MARCOS-GARCÍA, 1985).

Los ejemplares se capturaron en el mes de junio sobre umbelíferas en los subsectores Picoeuropeano y Laciano-Narceense.

DISTRIBUCIÓN GEOGRÁFICA:

Centroeuropea.

Material estudiado: 9c, 13-VI-86, 10. 30, 5-VI-88, 200 y $20^{\circ}$.

Cheilosia pubera (Zetterstedt, 1838)

Especie sólo conocida en la Península Ibérica del Pirineo catalán, de donde se citó por primera y única vez hace 60 años.

Ha sido colectada en el nivel subalpino en los subsectores LacianoNarceense y Ubiñense sobre Caltha palustris L., especie vegetal relacionada en Inglaterra con este sírfido.

DISTRIBUCIÓN GEOGRÁFICA:

Europa Central y Septentrional.

Material estudiado: 5a, 1-VI-88, 10*. 23a, 3-VI-88, 1 q. 
Especie bien distribuida por el territorio español. Ha sido capturada en los meses de verano en altitudes que oscilan entre los 150 y 1.123 m.s.n.m. No se han obtenido datos de esta especie en el subsector Laciano-Ancarense. op. cit.).

La larva se desarrolla en distintas especies de hongos (STUBBS \& FALK,

DISTRIBUCIÓN GEOGRÁFICA:

Euroasiática.

Material estudiado: 12, 11-IX-87, 10̛. 31, 10-IX-88, 10 . 39, 14-VII-87, 1 q. Cheilosia soror (Zetterstedt, 1843)

Especie también estival ya citada de diversas provincias españolas. Se ha capturado sobre umbelíferas de altitudes inferiores a 700 m.s.n.m. pertenecientes al subsector Picoeuropeano.

DISTRIBUCIÓN GEOGRÁFICA:

Euroasiática.

Material estudiado: $30,10-\mathrm{IX}-88,10.33,5-\mathrm{VII}-86,10^{\circ}$ y 1 . .

\section{Grupo B (Ojos y genas con setas)}

Cheilosia barbata Loew, 1857

Especie capturada a finales de la primavera y primeros días del verano en localidades comprendidas entre los 600 y 1.250 m.s.n.m., aunque se distribuye preferentemente en el piso mesomontano.

Once de los quince ejemplares se han capturado sobre Heracleum spondy lium $\mathrm{L}$. y el resto volando entre vegetación herbácea.

Esta especie se encuentra representada en todos los subsectores fitogeográficos diferenciados, excepto en el más occidental, el Naviano-Ancarense.

DISTRIBUCIÓN GEOGRÁFICA:

Europa Central y Septentrional.

Material estudiado: 9d, 3-VII-87, 10 y $2 \% q .12 \mathrm{~b}, 12-\mathrm{VII}-87,3 \mathrm{or}^{\circ}$ y $2 \%$. 21d, 3-VII-87, Io'. 30, 21-VI-87, 2क्ष. 39 b, 16-VI-86, 1\%; 14-VII-88, 1\%. 40, $23-\mathrm{VI}-87,10^{\prime}$ y 1 \%.

\section{Cheilosia frontalis Loew, 1857}

Esta especie en España se conocía de localidades montanas de las provincias de Huesca y Cáceres. Con los presentes datos se cita por vez primera de la Cornisa Cantábrica.

Los ejemplares se han capturado en su mayoría en el mes de junio en altitudes comprendidas entre 1.100 y 1.562 m.s.n.m. y sobre las siguientes plantas: 
Caltha palustris L. ................. 2 ơ y 1 q

Chaerophyllium aureum L. ........ $1 \sigma^{\circ}$

Ranunculus sp. ..................... $10^{*}$

Se han capturado representantes de esta especie en los subsectores LacianoNarceense, Ubiñense y Altocarrionés.

DISTRIBUCIÓN GEOGRÁFICA:

Euroasiática.

Material estudiado: 4b, 1-VI-88, 10'. 7, 1-VI-88, 10'. 13, 2-VI-88, 10 . 21c, 3-VI-88, 10" y 10. 36a, 6-VI-88, 10". 43, 21-VI-85, $10^{\circ}$.

Cheilosia illustrata (Harris, 1776)

En España, esta especie sólo se conocía del Pirineo Altoaragonés.

Se ha capturado en altitudes comprendidas entre los 500 y los 1.225 m.s.n.m. y en localidades situadas en los subsectores Laciano-Narceense, Ubiñense y Altocarrionés.

Once de los catorce ejemplares estudiados se han colectado sobre Heracleum spondylium L., planta de la que se alimentan las larvas. Este dato además coincide con lo expresado por GOELDLIN, 1974, quien indica que todas sus capturas han sido realizadas sobre umbelíferas y también en los meses de junio y julio.

En la misma planta coincidieron en el momento de la captura ejemplares de Psithyrus bohemicus (Seid.) (Hymenóptera), especie con la que Ch. illustrata presenta un claro mimetismo batesiano.

DISTRIBUCIÓN GEOGRÁFICA:

Euroasiática.

Material estudiado: 7, 3-VII-87, 10*. 9d, 3-VII-87, 10 . 12b, 5-VII-87, 10* y $5 \circ Q$. 34, 22-VI-87, 2oㅇ. 39b, 14-VII-87, 10’. 40, 23-VI-87, 10'. 45, 17-VII-85, 1\%. 46,18 -VII- 85,1 ,

Cheilosia intonsa Loew, 1857

Especie distribuida por distintos puntos peninsulares y localizada en la mitad oriental de la provincia Orocantábrica en altitudes que oscilan entre 250 y 1.050 m.s.n.m.

Distribución geográfica: Paleártico occidental.

Material estudiado: 33, 10-IX-86, 1․ 40, 22-VII-86, 1 \%.

Cheilosia maroccana Becker, 1894

En España se conocía esta especie de Cádiz y Málaga. El dato expuesto aumenta considerablemente su distribución hacia puntos más septentrionales.

DISTRIBUCIÓN GEOGRÁFICA:

Iberomogrebí.

Material estudiado: 44, 4-VI-88, 10 . 
Esta especie, aunque ya citada de distintas localidades de montaña distribuidas por el territorio español, es la primera vez que se cita para la Cornisa Cantábrica.

Se ha capturado entre los 600 y 1.432 m.s.n.m., mostrando preferencia por el nivel submontano. Se encuentra representada en todos los subsectores, excepto en el Naviano-Ancarense.

Han sido colectados 6 or $^{\circ}$ y 3 qQ sobre umbelíferas y 1 \& y 1 o sobre $R a$ nunculus repens, el resto mangueando sobre vegetación herbácea. Vuela principalmente en el mes de junio.

\section{DISTRIBUCIÓN GEOGRÁFICA:}

Eurosiberiana.

Material estudiado: 7, 1-VI-88, 10". 12, 14-VI-86, 1\%; 3-VI-87, 20"0; 5-VI87, 1․ 13, 2-VI-88, 10". 17, 4-VI-87, 1o. 19, 4-VI-87, 10'. 25, 6-VII-86, $10^{\circ}$. 29, 5-VI-88, 40'o'. 30, 21-VI-87, 1․ 38, 22-VI-87, 10'. 39, 22-VI-87, 1 \%.

Cheilosia vulpina (Meigen, 1822)

Especie sólo citada en la Península Ibérica de localidades prepirenaicas. Se ha capturado en el mes de junio en localidades montanas ubicadas en el subsector Ubiñense.

Los ejemplares fueron colectados sobre Ranunculus repens $\mathrm{L}$.

DISTRIBUCIÓN GEOGRÁFICA:

Centroeuropea.

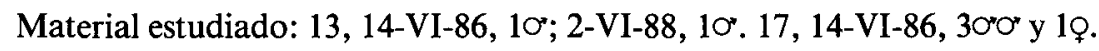

Grupo C (Ojos con setas, genas glabras, escudete sin macroquetas marginales)

Cheilosia alpina Zettersdet, 1838

Este ejemplar, capturado en un canchal silíceo, es el único representante de esta especie en el occidente europeo (MARCOS-GARCÍA, en prensa, a).

DISTRIBUCIÓN GEOGRÁFICA:

Alpes, Escandinavia y Siberia.

Material estudiado: 6c, 13-VI-86, $10^{\circ}$.

Cheilosia andalusiaca Torp, 1971

Esta especie, descrita de Sierra Nevada (TORP, 1971), ha sido posteriormente citada de Cáceres, León y Salamanca (MARCOS-GARCÍA, 1987). Ha sido siempre capturada en áreas de montaña, por encima de los 900 m.s.n.m. y sometidas a influencia atlántica.

DISTRIBUCIÓN GEOGRÁFICA:

Ibérica.

Material estudiado: 6c, 13-VI-86, 10". 
Especie sólo conocida en la Península Ibérica de Bilbao, Zaragoza y Barcelona, citándose ahora por vez primera de la Cordillera Cantábrica.

El ejemplar fue colectado a $1.310 \mathrm{~m}$. de altitud sobre Ranunculus repens $\mathrm{L}$.

DISTRIBUCIÓN GEOGRÁFICA:

Eurosiberiana.

Material estudiado: $17,4-\mathrm{VI}-87,10$.

Cheilosia chrysocoma (Meigen, 1822)

Estos ejemplares son los únicos que se conocen de esta especie en la $\mathrm{Pe}$ nínsula Ibérica (MARCOS-GARCÍA, en prensa, a).

Todos ellos han sido capturados en un prado silíceo subalpino sobre $R a$ nunculus repens en el subsector Naviano-Ancarense, contrastando este hecho con las condiciones típicas señaladas por STUBBS \& FALK, $o p$. cit., y que son los márgenes de bosques sobre suelos calizos en puntos siempre cercanos a condiciones pantanosas.

\section{DISTRIBUCIÓN GEOGRÁFICA:}

Europa Central y Septentrional.

Material estudiado: 1b, 12-VI-86, 300\%; 1-VI-87, 50\%

Cheilosia flavipes (Panzer, 1798)

Esta especie sólo se conocía en España de la Sierra de Guadarrama y del Prepirineo gerundés, por lo que los datos anteriores representan la primera cita para la Cordillera Cantábrica.

Los ejemplares se han cazado en el mes de junio sobre Caltha palustris L., en localidades situadas entre los 1.200 y 1.625 m.s.n.m. y pertenecientes al subsector Ubiñense.

\section{DISTRIBUCIÓN GEOGRÁFICA:}

Paleártico occidental.

Material estudiado: 21f, 3-VI-88, 1@. 23a, 16-VI-86, 20\%

Cheilosia limbicornis Strobl, 1909

Especie sólo conocida de Granada, por lo que el presente dato amplía considerablemente su distribución ibérica.

El ejemplar fue colectado a 1.225 m.s.n.m. sobre Ranunculus ollissiponensis Pers.

DISTRIBUCIÓN GEOGRÁFICA:

Ibérica.

Material estudiado: 9d, 1-VI-88, 1 \%. 
Cheilosia albitarsis (Meigen, 1822)

Esta especie, aunque se cita por vez primera para la Cordillera Cantábrica, ya se conocía de distintas localidades dispersas por el área peninsular ibérica.

Vuela principalmente en el mes de junio, ya que de los 66 ejemplares estudiados, sólo cinco se han capturado a principios del mes de julio.

Se han capturado ejemplares sobre:

Ranunculus repens L. .............. 5 \%ơ y 6 ф̊

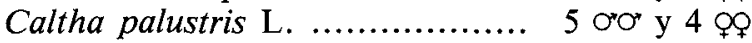

Ranunculus ollissiponensis Pers .. 6 ơ 0 y 8 ơ

Heracleum spondylium L. ......... 2 orơ y 8 ơ

Chaerophyllium aureum L. ....... 3 oq

Mangueando sobre vegetación her-

bácea ............................... 4 ơ y 15 q̊

Se han colectado ejemplares entre los 600 y 1.400 m. de altitud, mostrando preferencia por el piso bioclimático mesomontano.

Las capturas se han efectuado en los tres sectores definidos.

\section{DISTRIBUCIÓN GEOGRÁFICA:}

Paleártico occidental.

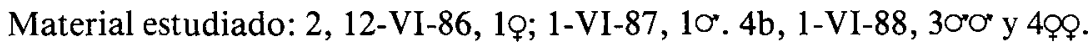

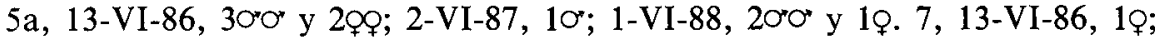
4-VII-87, 1; 1-VI-88, 1ㅇ. 9d, 3-VII-87, 3ㅇ; 1-VI-88, 60"0 y 8워. 13, 14-VI86, 1\%; 3-VI-87, 20\% y 6qo; 5-VII-87, 1ф; 2-VI-88, 10 y 3qo. 16b, 3-VI-87, 10'. 25, 6-VII-86, 1․ 29, 5-VI-88, 1o. 30, 21-VI-87, 10 y 1\%; 5-VI-88, 2qo. 35, 6-VI-88, 1 ㅇ.

\section{Cheilosia bergenstammi Becker, 1894}

Los ejemplares estudiados son los únicos conocidos de esta especie en la Península Ibérica (MARCOS-GARCÍA, en prensa, a).

Especie bivoltina (STUBBS \& FALK, op. cit.), capturada a finales de la primavera, pero principalmente a inicios del otoño, en diferentes altitudes comprendidas entre los 600 y 1.625 m.s.n.m., mostrando preferencia por zonas submontanas.

Los ejemplares fueron capturados sobre las siguientes especies vegetales:

Senecio aquaticus Hill. subsp. barbaraeifolius (Wim \& Grab.) Walters ..................................

En esta planta vive la larva, oca9 Ơం̛ y 7 ㅇํ sionando su marchitamiento (STUBBS \& FALK, op. cit.).

Caltha palustris L. ..................

Daucus carota L. .................... $10^{\circ}$

Mentha pulegium L. ............... 1 \& 
A excepción del ejemplar capturado en el Pto. de Pandetrave, los restantes corresponden a localidades pertenecientes al sector fitogeográfico UbiñensePicoeuropeano, siendo especialmente abundante en el Ubiñense.

DISTRIBUCIÓN GEOGRÁFICA:

Europa Central y Septentrional.

Material estudiado: 22b, 9-IX-88, 10 y 1o. 23a, 3-VI-88, 10 . 25a, 9-IX-

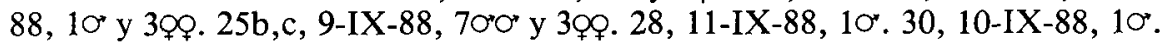
$36 \mathrm{a}, 6-\mathrm{VI}-88,1$.

Cheilosia correcta Becker, 1894

Especie hasta el momento sólo conocida de El Escorial (Madrid), de donde se citó por primera y única vez hace 60 años (GIL-COLLADO, op. cit.).

Los dos ejemplares se han capturado sobre Daucus carota L. en localidades inferiores a 1.000 m.s.n.m.

DISTRIBUCIÓN GEOGRÁFICA:

Centroeuropea.

Material estudiado: 3b, 4-VII-87, 1q. 33, 10-IX-88, 1 . .

Cheilosia fraterna (Meigen, 1830)

Especie capturada principalmente en el mes de junio, aunque algunas hembras han sido colectadas en los meses de verano. Estos individuos estivales pertenecen a la segunda generación parcial y estival a la que alude Rotheray.,, 1988.

Se encuentra preferentemente entre los 1.200 y $1.600 \mathrm{~m}$. y se haya representada en los subsectores Laciano-Narceense, Ubiñense y Altocarrionés.

DISTRIBUCIÓN GEOGRÁFICA:

Eurosiberiana.

Material estudiado: 5a, 13-VI-86, 2oq; 1-VI-88, 30*0 y 1o. 16b, 3-VI-87,

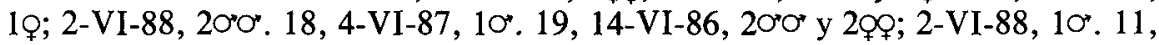
22-VI-87, 1Q. 21c, 3-VI-88, 20+ơ. 25, 6-VII-86, 4фᄋ; 12-IX-87, 1․ 35, 6-VI-88, 1ㅇ. 36d, 6-VI-88, $10^{\circ}$.

\section{Cheilosia gigantea (Zettersdet, 1843)}

En España, esta especie ya se conocía del Sistema Central, citándose ahora por vez primera del norte peninsular.

Se han capturado ejemplares en los meses de junio y septiembre, lo que hace suponer que se trata de una especie bivoltina. Se han obtenido datos desde los 50 a 1.550 m.s.n.m., mostrando cierta preferencia por el nivel submontano.

Se encuentra distribuida por todos los subsectores fitogeográficos.

Las plantas sobre las que se han realizado las capturas son:

Pteridium sp. ......................... 12 ơ 10 y 1 \%

Ranunculus sp. ...................... 8 ơ

Daucus carota L. .................... 2 \%ᄋ

Chaerophyllium aureum L. ........ 1 \% 


\section{DISTRIBUCIÓN GEOGRÁFICA:}

Eurosiberiana.

Material estudiado: 3a, 9-IX-87, 120 $0^{\circ}$. 4b, 1-VI-88, 1o. 5a, 2-VI-87, 10 y 2 oㅇ. 7, 1-VI-88, 1․ 8b, 1-VI-88, 10. 9d, 1-VI-88, 2o․ 13, 14-VI-86, 10; 2-VI-88, 60' . 30, 10-IX-88, 2क्. 31, 10-IX-88, 1Q. 32, 10-IX-88, 10". 40, 23-VI-87, 2qo.

\section{Cheilosia iberica Marcos \& Claussen, 1989}

Esta especie fue descrita junto con material procedente del Sistema Central español (MARCOS-GARCÍA \& CLAUSSEN, 1989).

Vuela en primavera. Los ejemplares han sido todos capturados sobre Chaerophyllium aureum $\mathrm{L}$.

\section{DISTRIBUCIÓN GEOGRÁFICA:}

Ibérica.

Material estudiado: 9, 1-VI-88, 200

Cheilosia impressa Loew, 1840

En España, esta especie ya estaba citada de Santander, Madrid y Huesca.

Se ha capturado mangueando sobre vegetación herbácea en altitudes comprendidas entre 150 y 1.550 m.s.n.m., aunque muestra una clara preferencia por altitudes inferiores a $800 \mathrm{~m}$.

Su período de vuelo se extiende desde finales de la primavera y durante el verano.

No se han obtenido datos de esta especie en el sector Altocarrionés.

\section{DISTRIBUCIÓN GEOGRÁFICA:}

\section{Euroasiática.}

Material estudiado: 3b, 4-VII-87, 10*. 5a, 2-VI-87, 10*. 9d, 3-VII-87, $10^{\circ}$. 21, 3-VII-87, 10; 9-IX-88, 10'. 26, 9-IX-88, 1‥ 27, 21-VI-87, 10 y 10. 30, 21VI-87, 10 y 1\%; 10-IX-88, 10' y 1‥ 31, 10-IX-88, 3ㅇ․

\section{Cheilosia mutabilis (Fallén, 1817)}

Esta especie ya estaba citada de distintas provincias españolas.

Se ha capturado en altitudes comprendidas entre los 250 y 1.562 m.s.n.m., en localidades situadas en todos los subsectores considerados, excepto en el más occidental: Naviano-Ancarense. Es una especie de finales de primavera y verano.

Las plantas sobre las que se han capturado ejemplares de esta especie son las siguientes:

Caltha palustris L. ................. 3 \&q

Tanacetum vulgare L. .............. 1 \&

Ranunculus sp. ...................... 1 o y 2 \%

Crataegus sp. .......................... $10^{\circ}$

Mentha sp. ......................... 1 o 


\section{DISTRIBUCIÓN GEOGRÁFICA:}

Paleártica occidental.

Material estudiado: 8b, 13-VI-86, 10. 9c, 13-VI-86, 10'. 12, 11-IX-87, $10^{\circ}$. 13, 25-VII-86, 1o. 19a, 25-VII-86, 1‥ 23a, 3-VI-88, 1ㅇ. 25a, 3-VI-88, 2фo. 27, 15-VI-86, 1o. 32c, 5-VII-86, 10'. 35, 6-VI-88, 10'. 36a, 6-VI-88, 1क. 36d, 6-VI86, 1․ 37b, 16-VI-86, 1ㅇ. 38, 16-VI-86, 10*. 44, 4-VI-88, 1 . .

Cheilosia praecox Zetterstedt, 1843

Esta especie se cita por vez primera en la Cordillera Cantábrica.

Los ejemplares se han capturado sobre ranunculáceas en altitudes comprendidas entre 1.000 y 1.700 m.s.n.m.

DISTRIBUCIÓN GEOGRÁFICA:

Paleártica.

Material estudiado: 6a, 13-VI-86, 10'. 28, 23-VII-85, 10".

Cheilosia proxima (Zetterstedt, 1843)

Es el único ejemplar de esta especie conocido en el ámbito peninsular ibérico (MARCOS-GARCÍA, en prensa, a).

DISTRIBUCIÓN GEOGRÁFICA:

Euroasiática.

Material estudiado: $3 b, 4-V I I-87,1$,

Cheilosia ruralis (Meigen, 1822)

Especie sólo citada anteriormente de Madrid y Zaragoza.

Ha sido capturada en el mes de junio, siempre sobre Caltha palustris L. y en localidades situadas por encima de 1.200 m.s.n.m. y pertenecientes a los subsectores Picoeuropeano y Altocarrionés.

\section{DISTRIBUCIÓN GEOGRÁFICA:}

Paleártica.

Material estudiado: 21c, 3-VI-88, 10'. 23a, 3-VI-88, 10'. 36, 6-VI-88, 10' y 1 .

\section{Cheilosia vernalis (Fallén, 1817)}

Especie ya citada de distintas provincas españolas, aunque los presentes datos son los primeros que se conocen para la Cordillera Cantábrica.

Se han capturado ejemplares desde el mes de mayo al de noviembre en todos los niveles altitudinales, aunque parece distribuirse preferentemente en los pisos colino y submontano (inferiores a $800 \mathrm{~m}$.).

Se encuentra representada en todos los subsectores, excepto en el LacianoNarceense.

Los imagos han sido colectados sobre distintas plantas, utilizando diferentes especies de compuestas como dieta alimenticia en sus estadíos larvarios (STUBBS \& FALK, op. cit.). 


\section{DISTRIBUCIÓN GEOGRÁFICA:}

\section{Eurosiberiana.}

Material estudiado: 3c, 9-IX-87, 10 . 12, 11-IX-87, 10*. 19a, 2-VI-88, 10. 23a, 16-VI-86, 1o. 25, 12-IX-87, 10; 9-IX-88, 10. 26, 9-IX-88, 1․ 32, 10-IX-

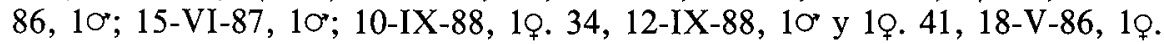

\section{Cheilosia zetterstedti Becker, 1894}

Especie conocida de diversas provincias españolas, aunque los datos aquí aportados son novedad para la Cordillera Cantábrica.

DISTRIBUCIÓN GEOGRÁFICA:

Europa Central y Septentrional.

Material estudiado: $42,10-I V-85,10^{\circ}$ y 1 .

\section{RESULTADOS Y DISCUSIÓN}

Los resultados faunísticos en la zona estudiada han sido de gran interés, ya que de un total de 354 ejemplares pertenecientes a 42 especies del género Cheilosia, sólo cuatro: Ch. nigripes, Ch. pagana, Ch. impressa y Ch. proxi$m a$, se conocían previamente de la Cordillera Cantábrica, siendo de destacar que en toda el área peninsular ibérica no se conocían más que 36 especies de este género.

Estas 42 especies representan el $24,7 \%$ del total de especies de la familia Syrphidae capturadas en dicha cordillera. Este alto porcentaje de representantes del género Cheilosia es también resaltado por CHEMINI \& al., 1986, en un estudio realizado en Monte Lessini (Trento, Italia), zona igualmente montañosa, con características subalpinas, donde el $30,4 \%$ de las especies examinadas pertenecían a este género.

Las larvas de Cheilosia, como se indicó previamente, presentan hábitos fitófagos; es, por tanto, lógico pensar que el elevado número de especies de este género holoártico presentes en la zona estudiada se deba, al menos en parte, a la riqueza florística y a la existencia de vegetación autóctona que presenta la provincia fitogeográfica Orocantábrica. Un ejemplo de ello son los bosquetes de abedules y hayas (relegados estos últimos a la franja norte peninsular y Sierra de Ayllón) que albergan entomofauna común a la de formaciones vegetales similares del resto de Europa.

Tres especies, Ch. gagatea, Ch. faucis y Ch. ahenea, se citan por vez primera en la Península Ibérica, siendo las dos primeras «raras» en la zona, ya que sólo se ha estudiado un espécimen de cada una. Por el contrario, $C h$. ahenea se encuentra bien representada en el área de estudio, en medios semejantes a donde se ha capturado en Centroeuropa.

Se confirma la presencia de $C h$. latifacies en la Península Ibérica, que había sido puesta en duda tras identificar como Ch. paralobi los ejemplares sobre los que se basaron las distintas citas de esta especie en España. Ambas especies son simpátridas.

El sector fitogeográfico con mayor número de especies de Cheilosia es el Laciano-Ancarense, situado en el extremo occidental de la Cordillera Cantá- 
brica. Destacan en este sector las estaciones con mayor número de especies de toda el área estudiada: Murias de Paredes (11 especies), Pto. Leitariegos ( 9 especies) y Laguna de Arbás (7 especies), pertenecientes a los niveles subalpino y altimontano. Es importante también resaltar el hecho de que los cuatro endemismos ibéricos presentes en nuestra área de estudio (dos de ellos nuevas especies para la ciencia) han sido exclusivamente capturados en dos de estas tres localidades mencionadas: dos en la Laguna de Arbás y otras dos en Murias de Paredes, ambas estaciones ubicadas en el subsector Laciano-Narceense. Esta riqueza de especies contrasta a priori con el hecho de que el sector LacianoAncarense es el que presenta una mayor homogeneidad de medios, siendo la mayoría de ellos pastizales abiertos de montaña, con escasa vegetación arbórea y arbustiva, que albergan, no obstante y de forma exclusiva, elementos endémicos, iberomogrebríes y boreoalpinos ausentes en el resto del área estudiada.

Es junio el mes en el que en la zona estudiada se alcanza el máximo de actividad de los imagos de las especies de Cheilosia, ya que 22 de las especies estudiadas vuelan exclusivamente en dicho mes, coincidiendo con los primeros días soleados tras la desaparición de la nieve, cuando la floración de las plantas asociadas con dichas especies es máxima.

Son especies bivoltinas, con un máximo en primavera y otro en otoño: Ch. bergenstammi, Ch. fraterna, Ch. gigantea y Ch. paralobi. Son, por el contrario, especies estivales: Ch. correcta, Ch. hercyniae, Ch. intonsa, Ch. illustrata, Ch. impressa, Ch. latifacies, Ch. mutabilis, Ch. pagana, Ch. scutellata y $C h$. soror, siendo $C h$. vernalis la especie que presenta un período de vuelo más dilatado, ya que se han capturado ejemplares desde la segunda quincena del mes de mayo hasta mediados de noviembre. Este hecho es debido a que poseen dos o más generaciones anuales (STUBBS \& FALK, op. cit.), cuyas larvas se alimentan de distintas especies de compuestas que se desarrollan en sucesivos períodos anuales.

La mayor parte de las especies de Cheilosia estudiadas son elementos eurosiberianos $(16,66 \%)$, euroasiáticos $(14,28 \%)$, centroeuropeos $(14,28 \%$ ) o centronoreuropeos $(14,28 \%)$. Cuatro de ellas son endemismos ibéricos, dos elementos iberomogrebíes y tres boreoalpinos.

\section{AGRADECIMIENTOS}

Quiero agradecer a C. Claussen, especialista alemán en el género Cheilosia, los múltiples intercambios de opiniones con él mantenidos mediante correspondencia y que han sido de tanta utilidad en la elaboración de este trabajo. 


\section{BIBLIOGRAFÍA}

CHEMINI, C., M. DACCORDI \& F. MASON. Ditteri Sirfidi di un bosco a Lárice (Larix decidua Mill.) dei Monte Lessini (Provincia di Trento). Studi trent. Sci. Nat., Acta biol., 57: 247-258.

DAGET, Ph., M. GODRON, et J. L. GUILLERM, M.1972: Profils écologiques et information mutuelie entre espéces et facteurs écologiques. Grund. Meth. Pflanz., Int. Symp. Veget. Rinteln, 1970: 121-149.

DAGET, PH. et M. GODRON, 1982. Analyse de l'écologie des espéces dans les communautés. Masson, Paris: 163 pp.

GIL-COLLADO, J., 1930. Monografía de los sírfidos de España. Trab. Mus. Nac. Cienc. Nat. Serie Zool., 54: 377 pp.

GOELDLIN DE TIEFENAU, P., 1974: Contribution à l'étude systématique et écologique des Syrphids (Diptera) de la Suisse occidentale. Mitt. Schweiz. Ent. Ges., 47: 151-252.

GOOT, V. S. van der, 1958. Quelques Syrphides (Dipt.) des Pyrénées et de la Sierra Nevada. Ent. Ber. Amst., 18: 93-96.

LUCAS, J. A. W., 1976. New species of the genus Pipizella Rondani, 1856 (Diptera, Syrphidae). Publ. Natuurhist. Genoot. Limburg, 26 (1-3): 5-16.

MARCOS-GARCÍA, M. ${ }^{2}$ A., 1985. Contribución al conocimiento de la sirfidofauna del Pirineo del Alto Aragón I (Diptera, Syrphidae). Bolm. Soc. Port. Ent. Sup. 1: 511-520.

- 1987. Descripción de la hembra de Cheilosia andalusiaca Torp, 1971. Mediterranea Ser. Biol., IX: 57-61.

- 1987. Sobre la presencia de Cheilosia latifacies Loew y Cheilosia paralobi Malski en la Peninsula Ibérica (Diptera, Syrphidae). Anales de Biología, XI: 99-101.

- En prensa, a. Nuevas especies de Cheilosia Meigen, 1822, para la entomofauna ibérica (Diptera, Syrphidae). Fragmenta Entomologica, XXI.

- En prensa, b. Cheilosia cantabrica n. sp. de la Península Ibérica (Diptera, Syrphidae). Nouv. Rev. Ent., V.

MARCOS GARCÍA, M. ${ }^{a}$ A., \& C. CLAUSSEN, 1989. Description of Cheilosia iberica, new species from the Iberian Peninsula (Diptera, Syrphidae). Bonn. Zool. Beitr., 40 (1): 57-62.

RIVAS-MARTÍNEZ, S., T. E. DÍAZ, J. A. F. PRIETO, J. LOIDI y A. PENAS, 1984. La vegetación de la Alta Montaña Cantábrica, Los Picos de Europa. Ediciones Leonesas, León, 295 pp.

ROTHERAY, G. E., 1988. Larval morphology and feeding patterns of four Cheilosia species (Diptera, Syrphidae) associated with Cirsium palustre L. Scopoli (Compositae) in Scotland. Journal of Natural History, 22, 17-25.

SACK, P., 1935. Die Fliegen der Paläarktischen Region (Syrphidae). E. Schw. Verl. Stuttgart, 4: $451 \mathrm{pp}$.

SPEIGHT, M. C. D. \& C. CLAUSSEN, 1987. Redefinition of Cheilosia ahenea and Ch. argentifrons with records extending the known range of these species in western Europa (Diptera, Syrphidae). Annls. Soc. Ent. Fr. (N. S.), 23 (3): 299-308.

STUBBS, A. E., \& S. J. FALK, 1983. British Hoverflies. Br. Ent. Nat. Hist. Soc. 253 pp.

THOMPSON, F. C., \& E. TORP, 1986. Synopsis of the European species of Sphegina Meigen (Diptera, Syrphidae). Ent. Scand., 17: 235-269.

TORP, E., 1971. Some Syrphidae from Spain with descriptions of two new species (Insecta, Diptera). Steenstrupia Zool. Mus. Univ. Copenhagen (1): 229-245. 


\section{APÉNDICE I}

\section{SECTOR LACIANO-ANCARENSE}

\begin{tabular}{|c|c|c|c|c|c|}
\hline LOCALIDAD & N. ${ }^{\circ}$ & U.T.M. & ALTITUD & PROVINCIA & PAISAJE \\
\hline Pto. Ancares ............ & 1 & 29TPH7949 & 1.648 & León & $\begin{array}{l}\text { Abedular (a) } \\
\text { Brezal (b) }\end{array}$ \\
\hline Pereda de Ancares .... & 2 & 29 TPH 8443 & 900 & León & Castañar \\
\hline Candín.............. & 3 & 29TPH8642 & 875 & León & $\begin{array}{l}\text { Piornal (a) } \\
\text { Sauceda (b) } \\
\text { Prado siega (c) } \\
\text { Robledal (d) }\end{array}$ \\
\hline Caboalles de Abajo .. & 4 & 29TQH1261 & 1.300 & León & $\begin{array}{l}\text { Brezal (a) } \\
\text { Prado siega (b) }\end{array}$ \\
\hline Pto. Leitariegos ....... & 5 & 29TQH1162 & 1.550 & León & $\begin{array}{l}\text { Prado diente (a) } \\
\text { Piornal (b) }\end{array}$ \\
\hline Laguna de Arbás ..... & 6 & 29TQH0963 & 1.690 & Asturias & $\begin{array}{l}\text { Prado diente (a) } \\
\text { Canchal (c) }\end{array}$ \\
\hline Villar de Santiago .... & 7 & 29TQH2555 & 1.100 & León & Melojar \\
\hline Pto. de la Magdalena & 8 & 29TQH 2850 & 1.425 & León & $\begin{array}{l}\text { Prado diente (a) } \\
\text { Piornal (b) }\end{array}$ \\
\hline Murias de Paredes .... & 9 & 29TQH2948 & 1.225 & León & $\begin{array}{l}\text { Piornal (a) } \\
\text { Abedular (b) } \\
\text { Sauceda (c) } \\
\text { Prado siega (d) }\end{array}$ \\
\hline
\end{tabular}

\section{SECTOR UBIÑENSE-PICOEUROPEANO}

\begin{tabular}{|c|c|c|c|c|c|}
\hline LOCALIDAD & N. ${ }^{0}$ & U.T.M. & ALTITUD & PROVINCIA & PAISAJE \\
\hline Mirantes de Luna ..... & 10 & 30TTN6751 & 1.150 & León & Sabinar \\
\hline Beberinos ............... & 12 & $30 \mathrm{TTN} 8150$ & 1.000 & León & $\begin{array}{l}\text { Prado siega (a) } \\
\text { Sauceda (b) } \\
\text { Canchal (c) }\end{array}$ \\
\hline Geras $. . . \ldots \ldots \ldots \ldots . . . . . .$. & 13 & $30 \mathrm{TTN} 7451$ & 1.100 & León & Hayedo \\
\hline Geras $\ldots \ldots \ldots \ldots \ldots \ldots \ldots$ & 14 & 30 TTN7352 & 1.300 & León & Canchal \\
\hline Alto de Aralla .......... & 15 & 30TTN7054 & 1.536 & León & Prado diente \\
\hline Aralla & 16 & 30TTN7055 & 1.400 & León & $\begin{array}{l}\text { Brezal silíceo (a) } \\
\text { Aulagar calizo (b) }\end{array}$ \\
\hline Valle de Casares ....... & 17 & 30 TTN7658 & 1.310 & León & $\begin{array}{l}\text { Prado siega (a) } \\
\text { Avellanar (b) }\end{array}$ \\
\hline Pto. Pajares ............ & 18 & 30TTN7563 & 1.379 & León & Prado \\
\hline Brañillín ................. & 19 & 30 TTN7563 & 1.379 & León & $\begin{array}{l}\text { Brezal-Tojal (a) } \\
\text { Abedular (b) }\end{array}$ \\
\hline Villamanín & 20 & 30 TTN8356 & 1.120 & León & Brezal \\
\hline
\end{tabular}


SECTOR UBIÑENSE-PICOEUROPEANO

\begin{tabular}{|c|c|c|c|c|c|}
\hline LOCALIDAD & $\mathrm{N}^{0}$ & U.T.M. & ALTITUD & PROVINCIA & PAISAJE \\
\hline 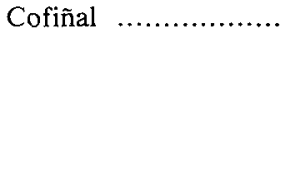 & 21 & $30 T U N 1468$ & 1.250 & León & $\begin{array}{l}\text { Brezal-Tojal (a) } \\
\text { Piornal (b) } \\
\text { Piñar-Abedular (c) } \\
\text { Turbera (d) } \\
\text { Hayedo calizo (e) } \\
\text { Prado diente (f) }\end{array}$ \\
\hline Cofiñal $\quad \ldots \ldots \ldots \ldots \ldots \ldots$ & 22 & $30 T U N 1470$ & 1.450 & León & $\begin{array}{l}\text { Aulagar (a) } \\
\text { Hayedo silíceo (b) }\end{array}$ \\
\hline Pto. de las Señales ... & 23 & 30TUN1771 & 1.625 & León & Prado diente \\
\hline Crémenes ................ & 24 & 30TUN2552 & 997 & León & Sabinar \\
\hline Alto del Pontón ........ & 25 & 30TUN3574 & 1.280 & León & $\begin{array}{l}\text { Hayedo (a) } \\
\text { Piornal (c) } \\
\text { Brezal (b) }\end{array}$ \\
\hline Santillán $\ldots . . . . \ldots \ldots \ldots \ldots$ & 26 & 30 TUN2693 & 150 & Asturias & $\begin{array}{l}\text { Sauceda (a) } \\
\text { Prado siega (b) }\end{array}$ \\
\hline Poncebos ................. & 27 & 30TUN5291 & 300 & Asturias & Encinar \\
\hline Fuente De ................ & 28 & 30 TUN5278 & 1.100 & Cantabria & $\begin{array}{l}\text { Hayedo (a) } \\
\text { Pastizal (b) }\end{array}$ \\
\hline Sotres $\ldots \ldots \ldots \ldots \ldots \ldots$ & 29 & 30TUN5888 & 1.025 & Asturias & Prado \\
\hline Tielve $\ldots \ldots \ldots \ldots \ldots \ldots$ & 30 & 30TUN5691 & 600 & Asturias & Pastizal \\
\hline Arenas de Cabrales ... & 31 & $30 T U N 5395$ & 150 & Asturias & Castañar \\
\hline Desf. de la Hermida & 32 & $30 T U N 6090$ & 250 & Cantabria & $\begin{array}{l}\text { Canchal (a) } \\
\text { Sauceda (b) } \\
\text { Prado siega (c) }\end{array}$ \\
\hline Lebeña $\ldots . .$. & 33 & 30TUN7185 & 300 & Cantabria & $\begin{array}{l}\text { Encinar (a) } \\
\text { Aulagar (b) }\end{array}$ \\
\hline
\end{tabular}

\section{SECTOR CAMPURRIANO-CARRIONÉS}

\begin{tabular}{|c|c|c|c|c|c|}
\hline LOCALIDAD & $\mathrm{N} \cdot{ }^{\circ}$ & U.T.M. & ALTITUD & PROVINCIA & PAISAJE \\
\hline Vada................. & 34 & 30TUN6472 & 600 & Cantabria & Robledal \\
\hline 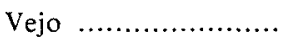 & 35 & 30 TUN6072 & 1.000 & Cantabria & Hayedo \\
\hline Pto. San Glorio ........ & 36 & 30TUN5669 & 1.609 & León & $\begin{array}{l}\text { Prado subalpino (d) } \\
\text { Piornal (b) }\end{array}$ \\
\hline Pto. de Pandetrave ... & $36 \mathrm{a}$ & 30TUN4874 & 1.562 & León & Hayedo \\
\hline Pedrosa del Rey ....... & 37 & 30TUN3959 & 1.100 & León & $\begin{array}{l}\text { Pinar (a) } \\
\text { Pastizal (b) }\end{array}$ \\
\hline Pto. del Pando ......... & 38 & 30TUN4054 & 1.432 & León & $\begin{array}{l}\text { Brezal (a) } \\
\text { Piornal (b) } \\
\text { Hayedo (c) }\end{array}$ \\
\hline Prioro $\ldots \ldots \ldots \ldots \ldots \ldots$ & 39 & 30TUN3951 & 1.123 & León & $\begin{array}{l}\text { Pinar (a) } \\
\text { Sauceda (b) }\end{array}$ \\
\hline Morgovejo .............. & 40 & 30 TUN3838 & 1.050 & León & Robledal \\
\hline
\end{tabular}


LOCALIDADES NO MUESTREADAS PERIÓDICAMENTE

\begin{tabular}{|c|c|c|c|c|c|}
\hline LOCALIDAD & N. ${ }^{\circ}$ & U.T.M. & ALTITUD & PROVINCIA & SUBSECTOR \\
\hline Balouta & 41 & 29ТРН7951 & 1.100 & Lugo & Laciano-Ancarense \\
\hline Cabañaquinta & 42 & $30 \mathrm{TTN} 8782$ & 500 & Asturias & Ovetense \\
\hline Piedrasluengas ......... & 43 & 30 TUN8166 & 1.280 & Palencia & Altocampurriano \\
\hline Prío, Unquera ......... & 44 & 30TUP7902 & 100 & Cantabria & Ovetense \\
\hline $\begin{array}{l}\text { San Salvador de Can- } \\
\text { tamuda } \ldots \ldots \ldots \ldots \ldots . . . . . . .\end{array}$ & 45 & 30TUN7858 & 1.100 & Palencia & Altocampurriano \\
\hline Vañes & 46 & 30TUN7852 & 1.100 & Palencia & Altocampurriano \\
\hline
\end{tabular}

Apéndice I.-Relación de localidades estudiadas.

Appendix I.-Studied localities. 
APÉNDICE II

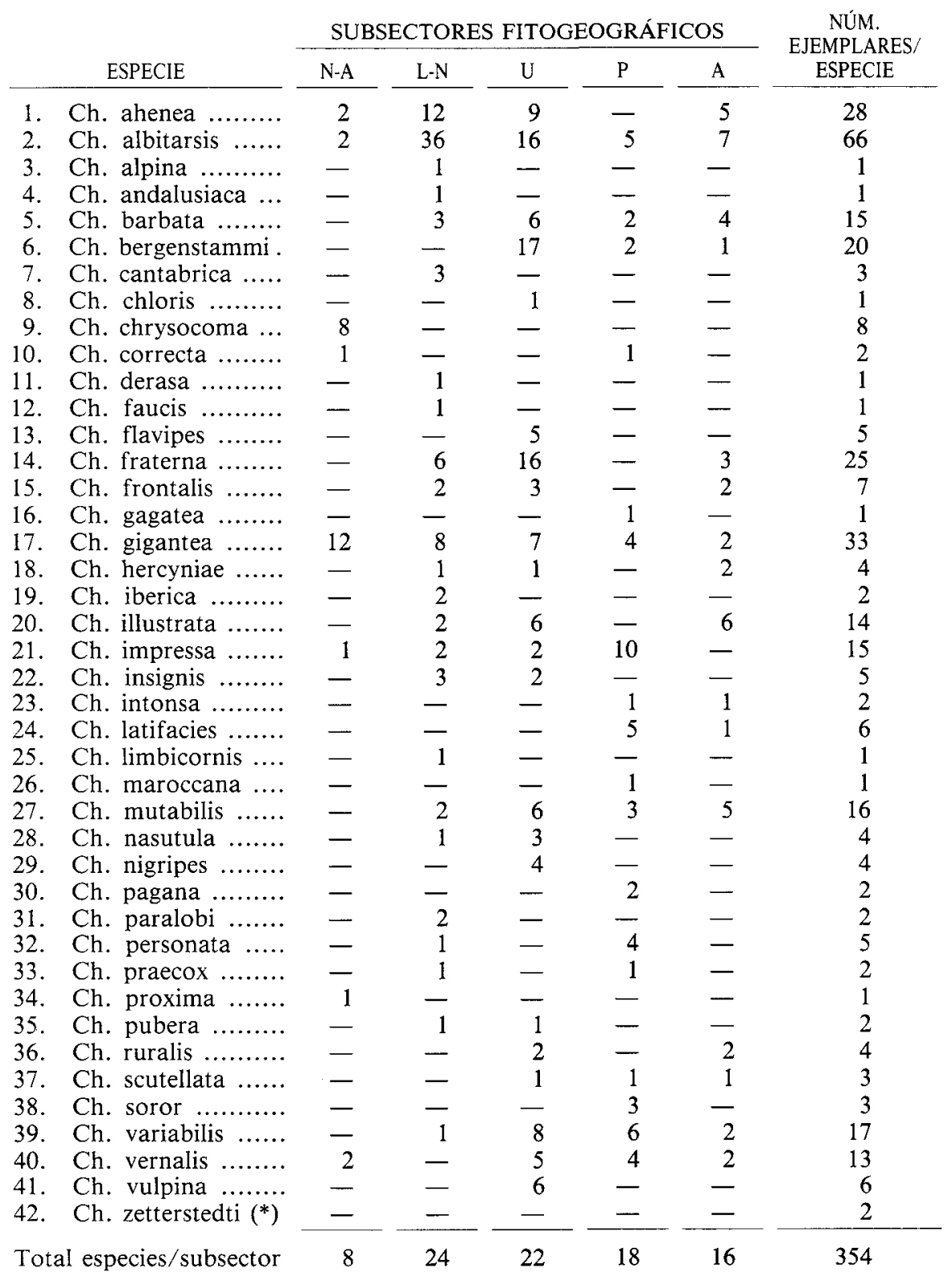

$\mathrm{N}-\mathrm{A}=$ Naviano-Ancarense. $\mathrm{L}-\mathrm{N}=$ Laciano-Narceense. $\mathrm{U}=$ Ubiñense. $\mathrm{P}=$ Picoeuropeano. $\mathrm{A}=$ Altocarrionés.

(*) Los dos ejemplares se capturaron en el subsector Ovetense (no considerados en nuestro estudio).

Apéndice II.-Especies estudiadas de la familia Syrphidae y relación de capturas en cada uno de los subsectores.

Appendix II.-Studied species of the family Syrphidae and their relation with the phytogeographic subsectors. 